Neil Selwyn

Monash University, Melbourne (Australie)

\section{Approches critiques des technologies en éducation : un aperçu'}

doi: 10.18162/fp.2019.579

\section{$\mathrm{R}$ ésumé}

Les technologies de l'information et de la communication constituent un élément central des services éducatifs et des pratiques pédagogiques

de l'époque contemporaine. Les institutions éducatives sont submergées de systèmes et d'outils numériques, alors que les individus réalisent de plus en plus fréquemment des parcours éducatifs et des apprentissages médiatisés (partiellement ou en totalité) par les technologies. Surtout, les technologies en éducation représentent aujourd'hui une industrie de plusieurs milliards de dollars et impliquent un nombre de plus en plus grand d'entreprises internationales au sein des systèmes éducatifs nationaux. Dans ce contexte, les approches critiques des technologies

en éducation nous semblent plus pertinentes que jamais. Le présent article a pour objectif d'en dresser un portrait, en commençant par rappeler leur pertinence et leurs fondements théoriques. Nous donnons ensuite un aperçu des études qui s'y sont attachées par le passé, avant de présenter les enjeux critiques contemporains. Finalement, nous terminons en identifiant des perspectives futures pour les approches critiques des technologies en éducation.

\section{Mots-clés}

Approches critiques, éducation, technologies, numérique.

\section{Introduction}

Les technologies de l'information et de la communication (désormais, «technologies ») font partie intégrante de l'éducation. Nous vivons en effet à une époque où les institutions éducatives, que ce soit les écoles, les universités, les bibliothèques ou les musées, sont submergées de systèmes et d'outils numériques. Les technologies viennent en appui aux moyens formels et informels par lesquels les individus s'engagent dans leur éducation - en particulier, les moyens par lesquels ils communiquent et interagissent, accèdent à l'information et génèrent des connaissances. Surtout, les technologies en éducation représentent une industrie de plusieurs milliards de dollars, dans laquelle les intérêts des entreprises technologiques internationales sont maintenant intimement liés aux pratiques et aux services éducatifs locaux.

Dans ce contexte, les chercheurs se doivent de développer une compréhension fine des technologies en éducation qui soit aussi englobante et réaliste que possible. Ce domaine n'a pas vocation à suivre un développement unidirectionnel susceptible de déboucher sur des consensus clairs et des vérités incontestables. Au contraire, il nécessite plutôt des examens, des remises en cause, des débats et des délibérations. Comme plusieurs l'ont fait valoir, il est grand temps pour les chercheurs étudiant les technologies en éducation de s'engager dans des discussions complexes et de s'attaquer aux enjeux critiques qu'elles soulèvent.

Toutefois, la majorité des productions scientifiques sur les technologies en éducation, que ce soit en termes de recherches ou de publications, négligent ces questions. Les chercheurs se montrent réticents à l'idée de se pencher sur les contradictions et l'inefficacité qui ont historiquement accompagné les tentatives d'intégration des technologies en éducation, préférant plutôt se concentrer sur les 
usages potentiels des technologies, ceux à venir. Il en résulte une préférence marquée pour l'état de l'art plutôt que l'état de la situation ${ }^{2}$. En résumé, il est possible de penser que les études sur les technologies en éducation pourraient faire mieux qu'elles ne font actuellement.

Ce manque de "mordant» dans l'étude des technologies en éducation s'inscrit dans une tendance générale à aborder ces dernières de manière trop simpliste. Plus de 50 ans après les premières expérimentations du Computer Tutor et du Mighty Micro, les discours éducatifs et scientifiques sur les technologies en éducation continuent d'être inlassablement teintés d'utopies ou de dystopies. Les discours réalistes et objectifs, pour leur part, sont souvent minés par le désir, bien compréhensible par ailleurs, d'améliorer l'éducation par tous les moyens possibles. Ce faisant, les technologies en éducation tendent à être abordées de manière grossière et conservatrice. Par exemple, l'importance de « réformer l'éducation en fonction des mutations technologiques et démographiques " (Institute of Directors, 2016, p. 8, traduction libre) est répétée ad infinitum par les décideurs politiques et les entrepreneurs, qui ne se penchent même pas sur les raisons pour lesquelles ces réformes éducatives devraient avoir lieu ni sur leurs implications pour les différents acteurs éducatifs.

Ainsi, les enjeux prédominants qui tendent à orienter le domaine des technologies en éducation sont réduits à savoir "Qu'est-ce qui fonctionne? ", voire "Qu'est-ce qui pourrait fonctionner? ». Confiner le territoire de questionnement sur les technologies en éducation à ce type d'enjeux est pour le moins insuffisant. L'étude des technologies en éducation ne peut pas se limiter à des enjeux d'efficacité ou de pratiques exemplaires. Il importe donc de s'interroger sur ces enjeux dominants, ne serait-ce que pour pousser plus loin la réflexion sur les bénéfices exacts que les technologies sont susceptibles d'apporter à l'éducation (et, a contrario, ceux qui ne dépendent pas d'elles). Dans cette perspective, le domaine des technologies en éducation a un besoin criant de plus de chercheurs et d'études critiques. La suite de cet article discute de certaines voies possibles dans cette direction en abordant les points suivants:

- Que signifie « être critique »?

- Les traditions critiques et les études précédentes

- Les études en cours sur les technologies en éducation

- Les pistes de recherche futures des études critiques sur les technologies en éducation

\section{Que signifie « être critique »?}

La critique des technologies a principalement suscité des réactions négatives lors des 20 dernières années. Cela s'explique - au moins en partie - par un inconfort envers la critique « destructive » qui a imprégné le tournant postmoderne des années 1990. Cette période a été marquée par des discours critiques complaisants et sans nuance envers les technologies, faite d' « ironie, de scepticisme, voire d'incrédulité paranoïaque » (Konstantinou, 2016, paragr. 3, traduction libre). Cela s'explique aussi par un retour de bâton contre la tendance, alimentée par les médias sociaux, d'une critique bruyante et sensationnaliste de la technologie pour des raisons d'audimat (Watson, 2016). En effet, les auteurs et analystes jouissent d'une attention considérable quand ils posent des questions comme «Est-ce que Google nous rend stupides? ", quand ils affirment que la génération actuelle est "La génération la plus stupide ", ou encore quand ils demandent " Comment les technologies du $21^{\mathrm{e}}$ siècle laissent des traces sur le cerveau ». Dans tous les cas, il s'agit de critiques simplistes et creuses formulées par des 
«critiqueux qui voient dans les technologies la perte de ce qui nous rend humains. Quelque part en chemin, les écrits critiques sur les technologies ont été assimilés à une méfiance réactionnaire envers le progrès » (Watson, 2016, paragr. 1-2, traduction libre).

Il est donc important de souligner que la position critique que nous défendons dans cet article ne correspond à aucune de ces tendances. Nous défendons plutôt une posture critique ayant du sens, une finalité, de l'objectivité et de la rigueur. Cela implique d'aborder les technologies avec méfiance et scepticisme - mais toujours dans un esprit constructif plutôt qu'avec cynisme. Pour préciser davantage notre posture critique, nous proposons cinq caractéristiques qui nous semblent mériter quelque approfondissement.

\section{Positionner les technologies comme un problème}

Les études critiques présentées dans cet article abordent les technologies en éducation comme étant problématiques. Comme l'avance Popkewitz (1987), le critère déterminant de la démarche critique est la tentative de "sortir des présuppositions et des pratiques de l'ordre établi et de s'efforcer de rendre problématiques les catégories, les présuppositions et les pratiques de la vie quotidienne » (p. 350, traduction libre). Il ne s'agit pas de stipuler que les technologies "sont le problème ", mais plutôt de reconnaître que leur intégration en éducation soulève des questions de première importance. Les approches critiques des technologies en éducation impliquent alors de produire des analyses détaillées et contextuellement riches, de procéder à une évaluation objective, et de prendre le temps d'examiner toutes les situations dans leurs côtés positifs, négatifs, et dans tous les intermédiaires possibles entre ces pôles. Elles exigent également d'être intrinsèquement sceptique tout en résistant à la tentation de tomber dans un cynisme stérile. En reprenant à notre compte une distinction faite par Foucault (1994), ceci implique de concevoir les technologies en éducation comme "dangereuses » plutôt que «mauvaises ", tout en gardant en tête que toute critique doit avoir l'action comme finalité, et non pas l'inertie :

Je ne cherche pas à dire que tout est mauvais, mais que tout est dangereux, ce qui n'est pas exactement la même chose que ce qui est mauvais. Si tout est dangereux, alors nous avons toujours quelque chose à faire. Donc ma position ne conduit pas à l'apathie, mais à un hypermilitantisme critique. Je crois que le choix éthico-politique que nous devons faire tous les jours, c'est déterminer quel est le principal danger. (p. 386, citation originale $\left.{ }^{3}\right)$

\section{Aborder les enjeux politiques et de pouvoir}

Les études critiques portent essentiellement sur la dimension politique des technologies en éducation et sur la façon dont elles participent de l'organisation et du déploiement du pouvoir. Rendre compte des technologies en éducation dans une posture critique consiste donc à reconnaître leur nature intrinsèquement politique. D'ailleurs, l'idée que les technologies ne sont pas neutres et qu'elles ont un caractère politique inhérent est bien établie dans la littérature scientifique (Winner, 1986). Ce faisant, les études critiques s'efforcent d'être aussi sensibles que possible aux enjeux de pouvoir, de contrôle, de conflit et de résistance qui accompagnent toute intégration des technologies en éducation. Autrement 
dit, les études critiques sur les technologies en éducation doivent être explicitement formulées en termes de relations potentiellement conflictuelles liées à la distribution du pouvoir. Ceci implique pour partie de considérer l'intégration des technologies en éducation comme une entreprise idéologique - c'està-dire comme un mécanisme clé permettant de promouvoir les valeurs et les intérêts hégémoniques dominants. Il est en effet nécessaire de décortiquer les dimensions idéologiques des technologies en éducation - c'est-à-dire de s'interroger sur les significations et les conceptions de l'éducation qu'elles véhiculent, de montrer comment elles manifestent les structures politiques et économiques, et de décrire comment des positions de pouvoir particulières sont développées, légitimées, reproduites et contestées.

\section{Mettre en évidence les silences et ce qui est réduit au silence}

En troisième lieu, les études critiques s'attachent aux désavantages, aux inégalités et aux injustices que les technologies suscitent en éducation. Comme le soutient Apple (2010), le rôle de tout chercheur travaillant dans le domaine de l'éducation devrait être d'agir en tant que «secrétaires critiques [...] des voix et des luttes de ceux qui, au quotidien, sont confrontés aux réalités de la vie dans des sociétés marquées par de profondes inégalités " (p. 97, traduction libre). En ce sens, il y a un besoin criant d'études reconnaissant le rôle que jouent les technologies dans la persistance des inégalités éducatives et dans l'établissement de nouvelles formes de division et de désavantage. Il s'agit ici de mettre en évidence les libertés et les « non-libertés » associées à l'intégration des technologies en éducation, en explorant comment les «bénéfices » supposés qu'elles proposent sont vécus par différents profils d'individus et de groupes sociaux. Plus important encore, il s'agit d'examiner dans quelle mesure les technologies renforcent et soutiennent les structures dominantes de production de pouvoir dans l'éducation.

\section{Viser à améliorer les choses}

Il est important de souligner que l'un des objectifs principaux des approches critiques est le désir de faire une différence. Plus précisément, leur finalité est de rendre l'intégration des technologies en éducation « meilleure » qu'elle ne l'est actuellement. Ainsi, adopter une posture critique des technologies en éducation est un exercice éminemment constructif. Les études critiques devraient donc viser à critiquer plutôt qu'à dénoncer - c'est-à-dire à « donner du sens aux choses, et non à les mettre en faute " (Watson, 2016, paragr. 22, traduction libre). En aucun cas, il ne suffit d'être « contre » les technologies plutôt que d'être « pour ». À l'aube des années 2020, il est insensé de nier l'existence des technologies - on ne peut être "contre " les technologies, pas plus qu'on ne peut être "contre " l'alimentation. Comme l'expliquent Bunz, Kaiser et Thiele (2012), toute critique des technologies doit être comprise comme un " geste affirmatif » - c'est-à-dire comme une prise de position sur les défaillances actuelles des technologies, tout proposant des formes renouvelées ou alternatives :

La critique en tant que geste affirmatif radical propose de s'attacher aux enjeux en question, de les manœuvrer et de les négocier dans la perspective d'un avenir " différent ", plutôt que de tenter de nous « séparer », de nous « distinguer » ou de nous « distancier» de ce qui ne nous plaît pas. (n. p., traduction libre) 


\section{Penser autrement}

Par souci d'objectivité, les chercheurs du domaine des technologies en éducation devraient toujours être ouverts à la possibilité que la meilleure solution de rechange à une situation donnée soit une solution qui n'implique aucun recours à la technologie, bien que l'idéal consiste plutôt à proposer différentes intégrations possibles des technologies. En ce sens, les chercheurs critiques devraient s'efforcer d'élaborer et de présenter un argumentaire convaincant démontrant de quelles manières et pour quelles finalités les technologies ont une place à prendre en éducation (Konstantinou, 2016). Il s'agit donc d'une démarche qui dépasse la documentation et l'interprétation de telle ou telle situation actuelle afin d'aboutir à une " orientation normative de ce qui devrait être en lieu et place de ce qui est " (Watson, 2016, paragr. 26, traduction libre). Comme le dit Livingstone (2012), un rôle important pour tout chercheur œuvrant dans le domaine des technologies en éducation est de demander « Comment les choses pourraient être autrement? » (p. 19, traduction libre). En réponse à cette question, il est possible de définir des "espaces de possibilité » permettant de résister, de perturber, de modifier et d'altérer. Le but ultime des approches critiques des technologies en éducation est donc d'utiliser leurs critiques et leurs discernements comme générateurs de stratégies alternatives. Ce faisant, il est important de rappeler qu'une critique n'a jamais «le dernier mot » sur une question donnée (Konstantinou, 2016, paragr. 10). Elle vise plutôt à ouvrir la conversation et à redoubler d'efforts dans la poursuite de meilleures façons de faire les choses.

\section{Traditions critiques et études précédentes}

Quels sont les fondements intellectuels des approches critiques? Il est important de noter qu'adopter une posture critique des technologies en éducation n'implique pas une adhésion dogmatique à une position théorique, à une école de pensée ou à un «-isme " particulier. Mais il est également important de noter qu'il existe un corpus substantiel d'écrits théoriques sur lequel s'appuyer pour articuler une posture critique.

En particulier, tous les points évoqués précédemment renvoient aux théories critiques qui ont été développées à la fin du $\mathrm{XIX}^{\mathrm{e}}$ siècle et au $\mathrm{XX}^{\mathrm{e}}$ siècle pour remettre en question le pouvoir, la domination et les inégalités qui ont accompagné l'ère industrielle. Ces traditions trouvent leur origine dans la critique du capitalisme de Marx. Les théories critiques peuvent donc être considérées comme une tentative d'actualiser les écrits de Marx aux développements ultérieurs du capitalisme managérial, de la bureaucratie étatique et de l'emprise croissante des corporations sur le travail et la vie sociale. Les préoccupations des théories critiques entrent clairement en résonance avec l'essor récent de la société numérique. Comme le soutient Berry (2014) :

Les théories critiques se sont toujours intéressées aux questions soulevées par les technologies [...]. Alors que les mutations technologiques se produisent à un rythme de plus en plus rapide - il est crucial que la littérature critique participe à ces [nouvelles] versions numériques [...]. Non pas que les théories critiques doivent être à l'affût de la dernière tendance numérique ou de la dernière mode d'internet, bien sûr, mais elles doivent par contre participer aux fondements et à la structuration des technologies. Ces derniers doivent être explorés dans leur matérialité et leurs 
affordances idéologiques, non seulement pour en offrir une analyse critique, mais aussi pour développer de nouveaux concepts et de nouvelles conceptions de ce monde numérique en flux continu. (p. 12, traduction libre)

Nous pouvons tout particulièrement souligner la contribution de l'École de Francfort - par exemple, Adorno, Habermas, Marcuse et Horkheimer - aux théories critiques. Ces théoriciens (et leurs collaborateurs) ont positionné les technologies comme un ensemble de processus et de pratiques éminemment politiques qui gagnent à être compris en termes de pouvoir et de contrôle. Ils ont mis l'accent sur les enjeux de domination via l'assujettissement institutionnel et le détournement de libertés démocratiques (plutôt que sur l'exploitation directe); les inégalités et l'injustice; les conflits et les résistances. Les théories critiques invitent à aborder les technologies en éducation de manière engagée, dans une dialectique du pouvoir technique faite de démocratisation et de totalisation potentielles. Ces approches soulignent l'intérêt d'identifier - puis de tester - la différence entre le potentiel et l'effectivité des technologies en éducation. Elles mettent également en évidence la nécessité de favoriser et d'appuyer les initiatives en faveur de l'autonomisation, de l'égalité, de la justice sociale et de la démocratie participative.

Un trait caractéristique des traditions critiques est de s'engager dans une «critique immanente ». Comme l'explique Antonio (1981), les théories critiques ne sont pas tant des théories générales que des démarches d'analyse. Elles visent à critiquer les principes qu'un système de valeurs dominant propose, et impliquent de relever les contradictions des processus et des logiques de production des formes dominantes de pouvoir et de contrôle. En tant que telle, la démarche du chercheur critique consiste à mettre en évidence les contradictions internes entre ce qu'une instance représente et ce qu'elle est et fait réellement. L'identification de contradictions ouvre sur des alternatives susceptibles de nous rapprocher un peu plus de la finalité ultime d'un changement social émancipateur.

Outre ces éléments spécifiques, plusieurs autres travaux théoriques peuvent être considérés comme contribuant aux théories critiques. Par exemple, on peut retirer beaucoup du travail de Foucault pour étudier la relation entre le pouvoir et les technologies. Dans ses écrits, Foucault a accordé une grande attention à la discipline, à l'(auto)régulation, à la gouvernementalité et aux relations entre savoir, pouvoir et contrôle social. Les travaux de Deleuze sur la nature interconnectée de la vie quotidienne et l'importance des affects ont également fait l'objet d'un intérêt grandissant au sein des théories critiques. Complémentairement, il existe un éventail de théories féministes, postcolonialistes, sociomatérialistes et post-humanistes qui, chacune à leur manière, peuvent permettre aux études sur les technologies en éducation d'aborder ces dernières dans une perspective avisée et sensible aux dimensions politiques.

Il est également important de souligner qu'il n'est pas nécessaire de réinventer la roue pour problématiser les enjeux qui accompagnent l'intégration des technologies en éducation (enjeux qui, d'ailleurs, sont rarement exclusifs à l'éducation et aux contextes éducatifs nationaux). De la même manière, il est important de rappeler que les études critiques actuelles s'inscrivent dans une tradition académique vieille de plusieurs décennies, développée par une communauté de chercheurs ayant abordé les technologies en éducation dans des perspectives similaires. En effet, bien qu'elle reste marginale par rapport aux courants dominants sur les technologies en éducation, une importante littérature scientifique produite dans les années 1980,1990 et 2000 offre un contrepoint critique. Les chercheurs qui sont probablement les plus connus dans la littérature anglophone incluent : 
- Postman (1992) et ses travaux sur les effets éducatifs limités de l'ordinateur, de la télévision et d'autres médias;

- Cuban et son analyse historique de l'intégration limitée des technologies dans les salles de classe américaines tout au long du XX ${ }^{\mathrm{e}}$ siècle (1988), ainsi que son analyse de la faible rentabilité ${ }^{4}$ des ordinateurs dans les écoles secondaires de la Silicon Valley (2001);

- Noble (1999) et son travail sur la déprofessionnalisation et la déqualification des enseignants de léducation supérieure par la formation à distance, qu'il a mémorablement appelé « Le moulin à diplômes numériques ${ }^{5}$;

- Roszak (1986), qui s'interroge sur le "Culte de l'information " croissant associé aux technologies en éducation;

- Bowers (1988) avec The cultural dimensions of educational computing: Understanding the nonneutrality of technology.

Cette période a aussi produit des ouvrages collectifs (p. ex., Beynon, Young et Mackay, 1991; Hlynka et Belland, 1991; Muffoletto, 2001), ainsi que des articles précurseurs de la part de chercheurs critiques importants en éducation (p. ex., Apple et Jungck, 1990; Bromley, 1997; Bryson et De Castell, 1998; Green et Bigum, 1993; Hodas, 1993; Kenway, Bigum, Fitzclarence, Collier et Tregenza, 1994; Kerr, 1996). Ces écrits antérieurs offrent un point de départ riche pour donner du sens aux orientations actuelles et futures des approches critiques sur les technologies en éducation, si fragmentés soientils. Par exemple, ces travaux ont été précieux pour mettre en lumière l'histoire complexe entre les technologies et l'éducation; ils ont également permis d'attirer l'attention sur des enjeux relatifs au travail enseignant (notamment dans la perspective de ce qu'on appelait dans les années 1980 une approche " néo-luddite »); ils ont aussi examiné l'économie politique des ordinateurs dans les écoles et les universités; et ils ont fait valoir la nature genrée de l'intégration des technologies en éducation et de ses effets. En prenant en considération le besoin actuel d'études critiques sur les technologies en éducation, il est important de faire bon usage de ce patrimoine scientifique.

\section{Portrait des études critiques actuelles sur les technologies en éducation}

Quel est l'état actuel des études critiques sur les technologies en éducation? Bien que la communauté de chercheurs critiques soit loin d'être cohérente et consensuelle, la quantité et la qualité des travaux critiques ont augmenté substantiellement depuis 2010. Ces travaux se situent pour la plupart en dehors des principales approches qui dominent le domaine des technologies en éducation, telles que les sciences cognitives, la formation des enseignants ou encore la psychopédagogie. En marge de ces approches dominantes, les chercheurs critiques œuvrent dans des champs d'études disparates, mais qui pointent tous, d'une manière ou d'une autre, dans la même direction (critique).

À titre d'exemple, les nouvelles littératies s'intéressent depuis longtemps aux technologies et aux textes numériques. Il en va de même pour l'éducation aux médias, la philosophie de l'éducation, les pédagogies critiques et l'éducation militante. Un foisonnement de travaux critiques des technologies en éducation voit également le jour au sein des études féministes, des études de genre et des études sur les politiques de l'éducation. Tous ces champs d'études mettent en lumière des enjeux scientifiques contemporains qui constituent autant de tendances actuelles des études critiques. Ces enjeux concernent entre autres les thématiques suivantes. 


\section{Les technologies en éducation et la reconfiguration de l'espace, du temps et de la responsabilité}

Un des enjeux « numériques » les plus importants ayant attiré l'attention des chercheurs critiques est l'expansion temporelle et spatiale des pratiques et des processus éducatifs au moyen des technologies. En effet, l'atténuation des frontières physiques est au cœur des promesses idéologiques des technologies en éducation. Les chercheurs critiques ont dépassé les préoccupations initiales formulées en termes de « compression du temps et de l'espace » ou de la « mort de la distance » pour explorer davantage les modalités et les implications de ces changements. Plus précisément, l'expansion et l'accélération de la formation à distance sur la base du "n'importe où, n'importe quand " soulève plusieurs questions sur la gestion de l'éducation et l'engagement des apprenants dans leurs études - ne serait-ce qu'en termes de répartition des responsabilités entre les différents sites et acteurs concernés (Hamilton, 2016; McLaren et Jandrić, 2015). Il est possible de penser que les technologies sont associées à une nouvelle distribution des opportunités éducatives et des obligations de retombées éducatives qui en résultent (Davies, 2016).

\section{Les technologies et l'(hyper)individualisation de l'éducation}

La section précédente rejoint les préoccupations que «l'individualisation » des parcours éducatifs via les technologies soulèvent chez les chercheurs critiques. La plupart des formules éducatives impliquant les technologies exigent un niveau de plus en plus élevé d'autonomie et d'esprit d'entreprise de la part des individus. Par conséquent, la réussite éducative dépend en premier lieu de la capacité des individus à autoréguler leur engagement dans leurs apprentissages au moyen des ressources pertinentes. De toute évidence, cette tendance éducative est généralement perçue comme étant favorable aux individus; pourtant, l'idée d'un apprenant autorégulé et autodéterminé qu'elle sous-tend met l'accent sur la capacité des individus à agir avec agentivité et empowerment. Sur ce constat, plusieurs chercheurs déconstruisent l'hypothèse bancale et peu convaincante d'un apprenant rationnel et individuel opérant au sein d'un environnement technologique efficace (Blum-Ross et Livingstone, 2016). Les études critiques sur le sujet laissent plutôt penser, dans le meilleur des cas, que seuls les apprenants de milieux privilégiés sont à même d'en tirer pleinement profit. Ainsi, l'individualisation qui accompagne ces modalités technologiques d'éducation peut être considérée comme autant de risques que d'opportunités pour les parcours éducatifs des individus.

\section{Les technologies et les inégalités éducatives}

Une préoccupation constante des chercheurs critiques est la persistance des inégalités et des injustices associées à l'intégration des technologies en éducation. En effet, encore récemment, des travaux indiquent que ces dernières continuent d'être différenciées sur plusieurs niveaux. Les inégalités numériques sont particulièrement marquées en fonction du statut socioéconomique, de l'origine sociale, de l'ethnie, du genre, du lieu de résidence, de l'âge et du niveau d'éducation (Cruz-Jesus, Vicente, Bacao et Oliveira, 2016; Dolan, 2015). Parallèlement aux inégalités d'accès, un consensus de plus en plus clair démontre que l'intégration des technologies en éducation n'est pas la solution équitable et démocratique qu'on lui attribue habituellement. Même lorsque les apprenants ont accès aux technologies, le type d'outils qu'ils utilisent, la manière dont ils les utilisent et les retombées qui en découlent sont médiés par une 
série d'inégalités numériques de "second niveau ». C'est, par exemple, clairement le cas dans les études critiques qui se penchent sur les disparités dans le suivi des massive open online courses (MOOCs) (p. ex., Allione et Stein, 2016; Knox, 2014). De telles études suggèrent qu'il est idéaliste et vain d'imaginer que les technologies sont nécessairement porteuses d'expériences éducatives démocratisées ou déségréguées.

\section{Les technologies et les contextes éducatifs}

Ces enjeux d'inégalités et de désavantages renvoient aux contextes dans lesquels les technologies sont adoptées et déployées. Un nombre grandissant de travaux s'intéresse à la manière dont les apprenants adoptent, redéfinissent et donnent un sens nouveau aux technologies à l'école, à l'université ou dans d'autres situations éducatives quotidiennes. Ces travaux soulignent aussi la prédominance de pratiques stabilisées dont les technologies en éducation font l'objet au niveau local. Sans prétendre à l'absence de changements, cela semble indiquer que les pratiques numériques sont en partie façonnées par le contexte - ne serait-ce que parce qu'elles sont toujours normalisées et routinisées dans des situations particulières.

Par exemple, un certain nombre d'études ont documenté l'usage ou le non-usage des technologies dans les écoles (p. ex., Peck et al., 2016; Philip et Garcia, 2015). Ces études fournissent une compréhension fine des logiques à partir desquelles les technologies sont incorporées dans un ensemble ${ }^{7}$ plus large de pratiques locales. Le portrait complexe qui en ressort met en lumière un certain nombre de déterminants relatifs à l'enseignant, au contexte scolaire composite de la salle de classe, de l'école, de la communauté locale et de l'état, ainsi que ceux relatifs à la présence de plusieurs options d'innovation concurrentes (Peck et al., 2016). Sur la base de ces études, il est important de garder en tête que les discours de changement et d'innovation qui tendent à accompagner les technologies en éducation sont compris et mis en œuvre de manière différente (et souvent divergente).

\section{L'éducation et la nature changeante du « travail » à l'ère du numérique}

Un petit nombre d'études ont abordé la manière dont les technologies en éducation constituent un site de travail, tant pour les enseignants et les apprenants, que pour les administrateurs. Ces études portent sur les processus de "main-d’œuvre numérique ${ }^{8}$ impulsés par les technologies au sein des institutions éducatives. À titre d'exemple, alors que les technologies sont de plus en plus utilisées pour partager, réutiliser à d'autres fins et externaliser des contenus pédagogiques, quelles sont leurs incidences sur la division accrue du travail et l'aliénation des enseignants vis-à-vis de leur enseignement (Hall,2015)? Les technologies sont aussi associées à un infléchissement croissant de distinctions binaires auparavant bien établies entre le travail et les loisirs, l'école et la maison, le temps d'études et le temps passé à s'occuper ${ }^{9}$ (Selwyn, Nemorin, Bulfin et Johnson, 2017). Les chercheurs critiques explorent également le rôle des technologies dans l'automatisation de l'activité scolaire, depuis les systèmes d'évaluation automatisée de tests et d'essais jusqu'aux systèmes d'apprentissage personnalisé qui régulent et individualisent le parcours de chaque étudiant, sans intervention de l'enseignant (Lundie, 2016; Thompson, 2016). Des questions du même ordre se posent au sujet de la surveillance et du contrôle via les technologies (Lindh et Nolin, 2016; Nemorin, 2017). Bien que ces « innovations » soient officiellement justifiées en termes d'efficacité accrue et de rationalisation, les études critiques permettent d'ouvrir sur des explications 
alternatives possibles dans lesquelles les technologies sont utilisées pour maintenir l'exploitation, la performance et l'aliénation.

\section{L'économie politique des technologies en éducation}

Comme mentionné précédemment, les chercheurs critiques appellent à "suivre le capital " lié à l'intégration croissante des technologies en éducation. Prenons par exemple la façon dont les technologies et l'impératif imaginaire du « digital » sont utilisés pour justifier la refonte, la réforme et la réorientation de la nature, de la forme et des valeurs de l'école publique. Des analyses critiques remettent en question les fondations philanthropiques, les corporations transnationales, les entreprises de placements à haut risque et autres «édu-preneurs » qui investissent de façon substantielle leur temps et leur argent afin de « réparer » et/ou de «transformer » nos systèmes éducatifs supposément «brisés " grâce à des solutions technologiques (Hogan, Sellar et Lingard, 2016). Ces dernières promettent d'implanter l'apprentissage personnalisé, la culture du faire ${ }^{10}$, les compétences du $21^{\mathrm{e}}$ siècle, etc. (Roberts-Mahoney, Means et Garrison, 2016; Selwyn et al., 2017). Elles proposent également de nouvelles visions de l'éducation dans la lignée de Altschooll ${ }^{11}$, Quest-to-Learn ${ }^{12}$ et Smarter Education d'IBM (Williamson, 2015). De plus en plus d'études critiques explorent l'intégration des technologies en éducation telle qu'elle est poussée par un « complexe d'industries éducatives » (Picciano et Spring, 2013, traduction libre) composé d'entreprises informatiques, de maisons d'éditions, de fondations et de think tanks et autres intérêts particuliers.

\section{Les technologies et la réforme néolibérale de l'éducation}

Dans la lignée de ces préoccupations se situent également des études critiques qui cherchent à décortiquer l'imbrication des technologies dans l'évolution de la gouvernance de l'éducation - notamment, comme moyens d'opérationnaliser les principes de performance, d'efficacité et d'imputabilité. À titre d'exemple, les chercheurs critiques s'interrogent sur divers systèmes en ligne qui permettent un examen public des écoles par la société civile, dans une logique de classement et de comparaison. C'est le cas, à l'échelle internationale, avec les bases de données de l'enquête PISA sur 65 pays de l'OCDE, ou encore, à l'échelle nationale, avec le site web My School du gouvernement australien (Sellar, 2014). Les études critiques s'intéressent également aux divers régimes de gouvernance numérique (p. ex., systèmes de modélisation algorithmique, de calcul et de recommandation) auxquels sont assujetties les institutions éducatives (Ozga, 2016). La plupart de ces développements sont dus à la place croissante accordée aux données numériques, ce qui soulève en retour des questions relatives à la datafication des systèmes et des institutions éducatives (Lingard, Sellar et Savage, 2014; Selwyn, 2015).

\section{Perspectives futures des approches critiques des technologies en éducation}

À l'heure où les technologies deviennent centrales dans les prestations, les processus et les pratiques éducatives, le besoin d'études critiques sur les technologies en éducation va croissant. Il est possible d'identifier au moins trois avenues qui pourraient contribuer à orienter les études critiques sur les technologies en éducation dans les prochaines années. 
Un premier développement probable est que les études critiques les plus pertinentes sur les technologies en éducation seront de plus en plus produites par des chercheurs qui ne se considèrent pas spécialistes de ce domaine. Récemment, les études critiques les plus éclairantes et perspicaces ont été menées par des chercheurs en sciences de l'éducation qui ont documenté des contextes éducatifs dans lesquels les technologies sont utilisées en abondance. De fait, dans la plupart des congrès en sciences de l'éducation (tel que le congrès annuel gigantesque de l'American Educational Research Association [AERA]), les meilleures analyses critiques des technologies ne sont pas issues des sessions, des symposiums ou des groupes d'intérêt particulier (SIG) sur les « technologies éducatives ». Elles sont plutôt éparpillées dans différentes sessions non spécifiquement dédiées aux technologies; mais elles n’en sont pas moins brillantes, bien quelles ne se réclament pas de ce domaine. Cette tendance se retrouve également dans les publications scientifiques, dans lesquelles les articles les plus éclairants sur les technologies en éducation sont davantage publiés dans des revues non dédiées aux technologies, telles que le Journal of Education Policy, Gender and Education ou encore Urban Education.

En deuxième lieu, il est possible que se développent des études critiques des technologies en éducation spécialisées dans des approches informatiques et computationnelles. Hors sciences de l'éducation, on assiste à une interpénétration croissante des sciences sociales appliquées aux technologies et des sciences computationnelles, ce dont témoignent la montée en puissance des Software Studies, des Data Studies et des Plateform Studies, qui sont autant de tentatives récentes de se pencher sur des questions critiques en sciences sociales par l'application de méthodes émanant des sciences computationnelles. Un domaine prometteur en sciences de l'éducation consisterait à documenter la manière dont sont programmées les technologies destinées à l'éducation. En effet, dans la mesure où une partie de l'activité éducative contemporaine se déroule en ligne dans des environnements numériques d'apprentissage ou autres, il y a tout lieu de s'intéresser davantage aux systèmes informatiques, aux environnements en ligne et aux espaces codés ${ }^{13}$ qui participent désormais de l'éducation. C'est d'ailleurs un point que les chercheurs des Software Studies et des Platform Studies ont déjà mis de l'avant. Comme le signale Manovich (2013), « le logiciel est devenu notre interface avec le monde, avec les autres, avec notre mémoire et notre imagination - un langage universel au travers duquel le monde s'exprime, et un moteur universel grâce auquel le monde fonctionne »(p. 2, traduction libre). Le besoin est donc réel de produire des études critiques qui explorent rigoureusement le code, les données et l'architecture des formes virtuelles contemporaines de l'éducation.

Troisièmement, des besoins de recherche se font sentir sur d'autres sujets que ceux couverts dans les deux premiers points ci-dessus. En effet, certains enjeux et tensions liés aux technologies en éducation méritent qu'on s'y penche au cours des prochaines années. À titre d'exemple, alors que l'éducation recourt de plus en plus à l'utilisation de l'intelligence artificielle, de données, d'algorithmes et d'infrastructures informatiques, plus d'études critiques sont nécessaires pour analyser la place grandissante occupée par les données au sein des institutions et des systèmes éducatifs - particulièrement en lien avec les enjeux de gouvernance, d'industrialisation et de vie privée. En outre, à l'heure où les technologies en éducation sont de plus en plus présentes à l'échelle mondiale, il est important de mener des études critiques sensibles aux cultures et aux contextes locaux et régionaux. Sur ce point, les études critiques sont particulièrement pertinentes pour prendre de la distance par rapport aux conceptions anglophones et/ ou eurocentriques des technologies en éducation, pour décoloniser ces dernières et remettre en cause les imaginaires hégémoniques américains poussés par la Silicon Valley. 
De plus, les chercheurs critiques gagnent aussi à anticiper l'essor des technologies post-numériques notamment, les applications éducatives des biotechnologies, des neuro-technologies et des technologies pharmaceutiques. Les avancées et la commercialisation des produits et des processus qui en découlent promettent d'être aussi importants durant la prochaine décennie que ne l'ont été les développements du micro-ordinateur et d'internet lors des quarante dernières années. Finalement, il importe de développer des études critiques dans des perspectives morales et éthiques, notamment en ce qui a trait à la production industrielle des technologies, qui pose des enjeux (trop peu discutés) d'exploitation de la main-d'œuvre et de dégradation de l'environnement naturel. Au même titre, l'impact environnemental dû à l'augmentation de la consommation d'énergie et des déchets électroniques fait implicitement partie du processus d'intégration des technologies en éducation. Les études critiques permettent de " sortir les technologies en éducation de leur bulle » et de les replacer dans leur contexte de production; celui d'un monde en crise, dans lequel elles ont des conséquences à long terme qui ne peuvent être ignorées.

\section{Conclusion}

Le présent article encourage fortement la poursuite des efforts de développement des études critiques des technologies en éducation. S'il ne fait aucun doute que les technologies «méritent toute notre attention et notre appréciation " (Watson, 2016, paragr. 10, traduction libre), force est de constater qu'elles méritent également scepticisme et doute systématique. À n’en pas douter, il s'agira toujours d'une position minoritaire, que beaucoup de chercheurs dans le domaine des technologies en éducation considèreront comme étant contre-intuitive, voire mal avisée. Être critique à l'égard des technologies constitue assurément une posture difficile à maintenir sur une longue période. Comme le souligne Lovink (2012), les études critiques sur les technologies passent souvent pour être pénibles, ingrates, ennuyeuses et peu attrayantes. Elles portent sur des sujets envers lesquels beaucoup d'éducateurs et de chercheurs en éducation sont indifférents et/ou hostiles, dans la mesure où ces sujets peuvent paraître décourageants. Il est d'ailleurs révélateur que plusieurs des grands noms des générations précédentes de chercheurs critiques qui sont mentionnés dans cet article aient abandonné les technologies en éducation pour œuvrer dans des domaines plus réceptifs aux approches critiques.

Toutefois, les travaux menés par les approches critiques ont toute leur raison d'être. Le défi pour ceux d'entre nous qui s'y inscrivent est de créer des espaces et des occasions à partir desquels l'on peut promouvoir un projet critique. Il est difficile - quoique pas impossible - d'obtenir des fonds de recherche dédiés spécifiquement à l'étude des enjeux décrits dans cet article. Pour autant, les organismes subventionnaires sont enclins à financer des projets qui proposent de nouvelles façons de penser et de mettre en ouvre les situations éducatives, ce qui rejoint les préoccupations centrales des études critiques. Pour cette raison, la réussite dans la structuration d'un programme critique dépendra en partie de l'état d'esprit dans lequel les études critiques seront menées. Les décideurs, les médias et les éducateurs sont volontiers ouverts aux analyses critiques si elles sont présentées de façon appropriée. En effet, comme l'a souligné cet article, les études critiques nont rien à gagner en adoptant un « ton moralisateur »(Massumi, 2015, p. 14, traduction libre). Elles ne gagnent pas non plus à adopter une position de surplomb, qui serait peu adéquate de surcroit. Les études critiques sur les technologies en éducation n'ont pas pour vocation d'offrir une meilleure perspective; elles permettent plutôt une 
perspective différente. Ainsi, les chercheurs critiques n'ont aucune bonne raison de mépriser les autres approches existantes ou de faire fi de celles-ci. Comme Felski (2015) le rappelle, le fait que d'autres études ne "soient pas critiques ne suffit à en faire des études acritiques » (p. 2, traduction libre). Dans cette perspective, si les études critiques sont conduites de façon constructive et dans un esprit d'ouverture, elles peuvent assurément trouver leur place dans le "firmament " des technologies en éducation, d'autant que le projet quelles proposent mérite d'être mené à bien. La balle est donc dans notre camp!

\section{Notes}

1 Ce texte est une production originale de Neil Selwyn initialement rédigée en anglais pour la revue Formation et profession dans le cadre du numéro thématique «Les théories critiques et le numérique en éducation : quelles propositions théoriques et quelles applications empiriques? ». Il a été traduit en français par Simon Collin et Alexandra H. Michaud de l'Université du Québec à Montréal.

2 "The "State of the Art" rather than the "State of the Actual" » dans la version initiale du texte.

3 La citation était en anglais dans la version initiale du texte. Elle a été remplacée lors de la traduction par la citation originale en français, tirée de Dits et écrits, 1954-1988, tome IV (1994), p. 386.

4 «"Oversold and underused" nature », dans la version anglaise du texte, en référence au titre du livre de Cuban (2001).

5 « Digital Diploma Mills » dans la version initiale du texte, en référence au titre du livre de Noble (1999).

6 « The Cult of Information », dans la version initiale du texte, en référence au titre du livre de Roszak (1986).

7 «Ecology », dans la version initiale du texte.

8 « Digital labor», dans la version initiale du texte.

9 «Productive work and busy-work », dans la version initiale du texte.

10 «Maker culture », dans la version initiale du texte.

11 Voir https://www.altitudelearning.com/ (en anglais).

12 Voir https://www.q21.org (en français).

13 «Coded spaces", dans la version initiale du texte.

\section{Références}

Allione, G. et Stein, R. M. (2016). Mass attrition: An analysis of drop out from principles of microeconomics MOOC. The Journal of Economic Education, 47(2), 174-186. http://dx.doi.org/10.1080/00220485.2016.1146096

Antonio, R. J. (1981). Immanent critique as the core of critical theory: its origins and developments in Hegel, Marx and contemporary thought. The British Journal of Sociology, 32(3), 330-345. http://dx.doi.org/10.2307/589281

Apple, M. W. (2010). Len Barton, critical education and the problem of 'decentred unities'. International Studies in Sociology of Education, 20(2), 93-107. http://dx.doi.org/10.1080/09620214.2010.503057

Apple, M. W. et Jungck, S. (1990). 'You don't have to be a teacher to teach this unit': Teaching, technology, and gender in the classroom. American Educational Research Journal, 27(2), 227-251. http://dx.doi.org/10.2307/1163008

Berry, D. M. (2014). Critical theory and the digital. Londres : Bloomsbury. http://dx.doi.org/10.5040/9781501302114

Beynon, J., Young, M. F. D. et Mackay, H. (1991). Understanding technology in education. Brighton : Falmer. 
Blum-Ross, A. et Livingstone, S. (2016). From youth voice to young entrepreneurs: the individualization of digital media and learning. Journal of Digital and Media Literacy. Repéré à http://eprints.lse.ac.uk/67045/

Bowers, C. (1988). The cultural dimensions of educational computing: understanding the non-neutrality of technology. New York, NY : Teachers College Press.

Bromley, H. (1997). The social chicken and the technological egg: Educational computing and the technology/society divide. Educational Theory, 47(1), 51-65. http://dx.doi.org/10.1111/j.1741-5446.1997.00051.x

Bryson, M. et De Castell, S. (1998). New technologies and the cultural ecology of primary schooling: Imagining teachers as Luddites in/deed. Educational Policy, 12(5), 542-567. http://dx.doi.org/10.1177/0895904898012005005

Bunz, M. (2012, 7-8 décembre). Facing our new monster: on critique in the era of affirmation. Terra Critica. Repéré à http://terracritica.net/wp-content/uploads/Bunz positionpaper.pdf

Bunz, M., Kaiser, B. M. et Thiele, K. (2012). What is critique in the $21^{\text {st }}$ century? Terra Critica. Repéré à https://www.academia.edu/7052525/What is Critique in the 21st century A conversation about Terra Critica the Interdisciplinary Network for Critical Humanities

Cruz-Jesus, F., Vicente, M. R., Bacao, F. et Oliveira, T. (2016). The education-related digital divide: An analysis for the EU28. Computers in Human Behavior, 56, 72-82. http://dx.doi.org/10.1016/j.chb.2015.11.027

Cuban, L. (1988). Teachers and machines: The classroom use of technology since 1920. New York, NY: Teachers College Press.

Cuban, L. (2001). Oversold and underused: Computers in the classroom. Cambridge, MA : Harvard University Press.

Davies, R. (2016). Ceaselessly exploring, arriving where we started and knowing it for the first time. Studies in Philosophy and Education, 35(3), 293-303. http://dx.doi.org/10.1007/s11217-016-9515-6

Dolan, J. E. (2015). Splicing the divide: A review of research on the evolving digital divide among K-12 students. Journal of Research on Technology in Education, 48(1), 16-37. http://dx.doi.org/10.1080/15391523.2015.1103147

Felski, R. (2015). The limits of critique. Chicago, IL : Chicago University Press. http://dx.doi.org/10.7208/chicago/9780226294179.001.0001

Foucault, M. (1994). Dits et écrits, 1954-1988, Tome IV. Paris : Gallimard. http://dx.doi.org/10.14375/np.9782070739899

Green, B. et Bigum, C. (1993). Aliens in the classroom. Australian Journal of Education, 37(2), 119-141. http://dx.doi.org/10.1177/000494419303700202

Hall, R. (2015). For a political economy of massive open online courses. Learning, Media and Technology, 40(3), 265-286. http://dx.doi.org/10.1080/17439884.2015.1015545

Hamilton, E. C. (2016). Technology and the politics of university reform: The social shaping of online education. New York, NY : Palgrave Macmillan. http://dx.doi.org/10.1057/9781137503510

Hlynka, D. et Belland, J. (1991). Paradigms regained: the uses of illuminative, semiotic and post-modern criticism as modes of inquiry in educational technology. Englewood Cliffs, NJ : Educational Technology Publications.

Hodas, S. (1993). Technology refusal and the organizational culture of schools. Education Policy Analysis Archives, 1(10), 197-218. http://dx.doi.org/10.14507/epaa.v1n10.1993

Hogan, A., Sellar, S. et Lingard, B. (2016). Commercialising comparison: Pearson puts the TLC in soft capitalism. Journal of Education Policy, 31(3), 243-258. http://dx.doi.org/10.1080/02680939.2015.1112922

Institute of Directors. (2016). Lifelong learning: Reforming education for an age of technological and demographical change. Repéré à https://www.iod.com/Portals/0/PDFs/Campaigns\%20and\%20Reports/Employment\%20and\%20Skills/Life\% 20Long\%20Learning\%20Report.pdf?ver=2016-09-14-124014-230

Kenway, J., Bigum, C., Fitzclarence, L., Collier, J. et Tregenza, K. (1994). New education in new times. Journal of Education Policy, 9(4), 317-333. http://dx.doi.org/10.1080/0268093940090403

Kerr, S. T. (1996). Toward a sociology of educational technology. Dans D. Jonassen (dir.), Handbook of research on educational technology (p. 113-142). Chicago, IL : Macmillan. 
Knox, J. (2014). Digital culture clash: 'massive' education in the E-learning and digital cultures MOOC. Distance Education, 35(2), 164-177. http://dx.doi.org/10.1080/01587919.2014.917704

Konstantinou, L. (2016, 17 juillet). The hangman of critique. Los Angeles Review of Books. Repéré à https://lareviewofbooks.org/article/the-hangman-of-critique/

Lindh, M. et Nolin, J. (2016). Information we collect: Surveillance and privacy in the implementation of Google Apps for Education. European Educational Research Journal, 15(6), 644-663. http://dx.doi.org/10.1177/1474904116654917

Lingard, B., Sellar, S. et Savage, G. C. (2014). Re-articulating social justice as equity in schooling policy: the effects of testing and data infrastructures. British Journal of Sociology of Education, 35(5), 710-730. http://dx.doi.org/10.1080/01425692.2014.919846

Livingstone, S. (2012). Critical reflections on the benefits of ICT in education. Oxford Review of Education, 38(1), 9-24. http://dx.doi.org/10.1080/03054985.2011.577938

Lovink, G. (2012). Networks without a cause: A critique of social media. Cambridge : Polity.

Lundie, D. (2016). Authority, autonomy and automation: the irreducibility of pedagogy to information transactions. Studies in Philosophy and Education, 35(3), 279-291. http://dx.doi.org/10.1007/s11217-016-9517-4

Manovich, L. (2013). Software takes command. New York, NY : Bloomsbury. http://dx.doi.org/10.5040/9781472544988

Massumi, B. (2015). Politics of affect. Cambridge : Wiley.

McLaren, P. et Jandrić, P. (2015). The critical challenge of networked learning: Using information technologies in the service of humanity. Dans P. Jandrić and D. Boras (dir.), Critical learning in digital networks (p. 199-226). Berlin : Springer. http://dx.doi.org/10.1007/978-3-319-13752-0 10

Muffoletto, R. (2001). Education E technology: critical and reflective practices. Cresskill, NJ : Hampton Press.

Nemorin, S. (2017). Post-panoptic pedagogies: the changing nature of school surveillance in the digital age. Surveillance and Society, 15(2), 239-253. http://dx.doi.org/10.24908/ss.v15i2.6033

Noble, D. F. (1999). Digital diploma mills. New York, NY : Monthly Review Press.

Ozga, J. (2016). Trust in numbers? Digital education governance and the inspection process. European Educational Research Journal, 15(1), 69-81. http://dx.doi.org/10.1177/1474904115616629

Peck, C., Hewitt, K. K., Mullen, C. A., Lashley, C. A., Eldridge, J. A. et Douglas, T. M. O. (2016). Digital youth in brick and mortar schools: Examining the complex interplay of students, technology, education, and change. Teachers College Record, 117(5), 1-40.

Philip, T. et Garcia, A. (2013). The importance of still teaching the iGeneration: New technologies and the centrality of pedagogy. Harvard Educational Review, 83(2), 300-319. http://dx.doi.org/10.17763/haer.83.2.w221368g1554u158

Philip, T. M. et Garcia, A. (2015). Schooling mobile phones: Assumptions about proximal benefits, the challenges of shifting meanings, and the politics of teaching. Educational Policy, 29(4), 676-707. http://dx.doi.org/10.1177/0895904813518105

Picciano, A. G. et Spring, J. (2013). The Great American education-industrial complex: Ideology, technology, and profit. Londres : Routledge. http://dx.doi.org/10.4324/9780203120613

Popkewitz, T. S. (1987). Critical studies in teacher education: its folklore, theory and practice. Brighton : Falmer.

Postman, N. (1992). Conscientious objections: Stirring up trouble about language, technology and education. Londres : Vintage.

Roberts-Mahoney, H., Means, A. J. et Garrison, M. J. (2016). Netflixing human capital development: personalized learning technology and the corporatization of K-12 education. Journal of Education Policy, 31(4), 405-420. http://dx.doi.org/10.1080/02680939.2015.1132774

Roszak, T. (1986). The cult of information: The folklore of the computer and the true art of thinking. New York, NY : Pantheon.

Sellar, S. (2014). Transparency and opacity: Levinasian reflections on accountability in Australian schooling. Educational Philosophy and Theory, 47(2), 118-132. http://dx.doi.org/10.1080/00131857.2013.793924 
Selwyn, N. (2015). Data entry: Towards the critical study of digital data and education. Learning, Media and Technology, 40(1), 64-82. http://dx.doi.org/10.1080/17439884.2014.921628

Selwyn, N., Nemorin, S., Bulfin, S. et Johnson, N. F. (2017). Everyday schooling in the digital age: High school, high tech? Oxford : Routledge.

Thompson, G. (2016). Computer adaptive testing, big data and algorithmic approaches to education. British Journal of Sociology of Education, 38(6), 827-840. http://dx.doi.org/10.1080/01425692.2016.1158640

Watson, S. M. (2016, 29 juin). How Virginia Heffernan is reinventing tech criticism. Columbia Journalism Revierw. Repéré à http://www.cjr.org/tow center/tech criticism virginia heffernan.php

Williamson, B. (2015). Educating the smart city: schooling smart citizens through computational urbanism. Big Data E Society, 2(2). http://dx.doi.org/10.1177/2053951715617783

Winner, L. (1986). The whale and the reactor: A search for limits in an age of high technology. Chicago, IL : University of Chicago Press.

\section{Pour citer cet article}

Selwin, N. (2018). Approches critiques des technologies en éducation : un aperçu. Formation et profession, 27(3), 6-21. http://dx.doi.org/10.18162/fp.2019.578 